\title{
(UN)PERSÖNLICHE STRUKTUREN IM LATEINISCHEN DER VULGATA UND IHRE HEBR ÄISCHEN PARALLELEN
}

\section{Chaja V. Duerrschnabel}

Universität Bern, Institut für Judaistik, Länggassstrasse 51, CH-3012 Bern, Switzerland vera.duerrschnabel@theol.unibe.ch

The phenomenon of impersonal verbs in Indo-European languages, particularly in Latin, is a much discussed issue. Nevertheless, the existence and use of impersonal verbs in the Latin of the Vulgate has never been investigated from a comparative point of view. The main aim of this article is to discuss the use of impersonal verbs in ancient Indo-European languages and Biblical Hebrew on the one hand, while on the other hand this article examines the translation of the impersonal verbs of Biblical Hebrew to the Latin of the Vulgate and its underlying principles.

Keywords: impersonal verbs, Vulgate, Hebrew, non-canonical argument structure.

\section{Verwendete Abkürzungen}

\begin{tabular}{|l|l|}
\hline 1. & 1. Person \\
\hline 2. & 2. Person \\
\hline 3. & 3. Person \\
\hline ADJ & Adjektiv \\
\hline AKK & nota accusativi \\
\hline ART & Artikel \\
\hline CAUS & Kausativ \\
\hline COP & Copula \\
\hline F & Feminin \\
\hline HIF & Hifil \\
\hline IMP & Imperativ \\
\hline M & Maskulin \\
\hline NEG & Negation \\
\hline NIF & Nifal \\
\hline DU & Dual \\
\hline PFV & Perfektiv \\
\hline POSS & Possessiv \\
\hline PRÄ & Präfixkonjugation \\
\hline PTCP & Partizip \\
\hline Q & Fragepartikel \\
\hline QAL & Qal \\
\hline SA & status absolutus \\
\hline SC & status constructus \\
\hline SG & Singular \\
\hline PL & Plural \\
\hline SUF & Suffixkonjugation \\
\hline WC & waw consecutivum \\
\hline
\end{tabular}

(C) St. Petersburg State University, 2017 


\section{Impersonalia im biblischen Latein}

Einige derjenigen Prädikate, die im klassischen Latein eine nicht-kanonische Markierung aufweisen, tendieren im biblischen Latein zu einer kanonischen Markierung. Der vorliegende Beitrag betrachtet ausgehend von den Impersonalia der alten indogermanischen Sprachen und dem Gebrauch unpersönlicher Konstruktionen im klassischen Hebräischen diese Prädikate genauer. Erstmals steht dabei die Übertragung unpersönlicher Strukturen aus dem Hebräischen in das Lateinische der Vulgata im Zentrum einer komparatistischen Untersuchung.

Für Nominativ-Akkusativ-Sprachen wie das Lateinische bedeutet eine kanonische Markierung, dass ein Satz mit zweiteiligem Prädikat eine transitive Struktur aufweist, und dass das primäre Argument des Satzes hinsichtlich Belebtheit, Agentivität, Definitheit oder Topikalität auch das grammatische Subjekt darstellt, welches unabhängig von seiner semantischen Rolle mit dem Nominativ markiert wird und die verbale Kongruenz auslöst. Das sekundäre Argument des Satzes wird hingegen bei einer kanonischen Markierung unabhängig von seiner semantischen Rolle mit dem Akkusativ kodiert und verursacht keine verbale Kongruenz (z. B.Dt. Anna sammelt Briefmarken). Bei einer nichtkanonischen Markierung wird hingegen das primäre Argument des Satzes mit einem obliquen Kasus markiert, während die morphosyntaktischen Merkmale des Subjekts einem anderen Nominal zugewiesen werden (z. B.Ihr gefallen Briefmarken). Prädikate mit nicht-kanonischer Markierung sind häufig Impersonalia, d. h. Verben, die keine Motivationsfähigkeit nach der grammatischen Person aufweisen, wobei jedoch zu beachten ist, dass es strictu sensu »einen formalen Unterschied zwischen echten Impersonalia und anderen Prädikaten nicht-kanonischer Strukturen [gibt]: während echte Impersonalia wie Lat. pluit oder Dt. mir ist kalt kein Subjekt haben, erscheint ein Subjekt in Strukturen wie mir gefällt etwas, wie man an der Variation Singular/Plural sehen kann (mir gefällt dieses Kleid vs. mir gefallen diese Kleider)« (Viti 2014, 154f.). Obgleich diese Differenz der argumentalen Struktur auch mit einem unterschiedlichen syntaktischen Verhältnis ${ }^{1}$ verbunden ist, können Impersonalia dennoch mit anderen nicht-kanonischen Strukturen betrachtet werden, da sie ebenfalls eine Degradierung des Agens ausdrücken. ${ }^{2}$

\section{Witterungsverben}

Bereits von Brugmann $(1904,625)$ werden Ausdrücke für Naturbeschreibungen als typisches Beispiel der Impersonalia betrachtet. Impersonale Witterungsverben sind seit dem Altlateinischen belegt, beispielsweise pluit und tonat bereits in der Sprache des Plautus (cf. Leumann/Hoffmann/Szantyr 1997, 415). Das Lateinische weist folgende unpersönlich gebrauchten Witterungsverben auf:" tonat »es donnert", pluit »es regnet", vesperascit »es wird Abend «, grandinat »es hagelt«, nivit /ninguit »es schneit«, fulget »es blitzt«, lucet »es ist

${ }^{1}$ Viti $(2014,155)$ verweist hier darauf, dass »in Konstruktionen mit Nominativ und Obliquus wie mir gefällt etwas $\langle\ldots\rangle$ auch der Obliquus bei einigen syntaktischen Tests wie Reflexivierung und Anhebung den Status des Subjekts zeigen kann, was für echte Impersonalia nicht der Fall ist.".

${ }^{2}$ Bereits von Priscian wurde die dritte Person als diejenige Person erkannt, die bei unpersönlichen Strukturen angewendet wird, inest igitur intellectu nominatiuus in ipsis uerbis, quo sine substantia significari non poterat, in prima quidem persona et secunda definitus, in tertia uero, quia innumerabiles sunt personae tertiae, infinitus, nisi excepta fiat actio, sicut fulminat, tonat; ea enim, quamuis non addamus nomen, definita esse uidentur, cum ad solum pertineant Iouem (Prisc. Inst. gramm. 3,116f.).

3 Die Aufzählung beruht auf Leumann/Hoffmann/Szantyr 1997, 415f. und Woodcock 1958, 166. 
hell«, fulminat »es blitzt«, rorat »es taut«, contenebrascit »es wird stockfinster «, lapidat »es regnet Steine «, disserenascit»es wird heiter «, hiemat»es herrscht Winter «. Der unpersönliche Gebrauch von Witterungsverben ist im gesamten indogermanischen Raum verbreitet: ${ }^{4}$

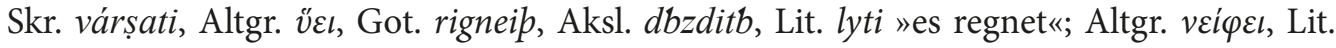
snigti »es schneit«; Skr. stanáyati, Ahd. donarot, Aksl. grbmbti, Lit. griáudeti »es donnert«; Lit. zaibuoiti »es blitzt«; Skr. tápati »es ist heiss«. Neben dem unpersönlichen Gebrauch der Witterungsverben, treten im Lateinischen auch persönlich konstruierte Strukturen auf, wobei zwischen denjenigen Strukturen mit explizitem Agens, und denjenigen, die lediglich ein Pronomen verwenden, unterschieden werden muss. Während Strukturen mit explizitem mit dem Nominativ markierten Agens wie Iupiter tonat "Zeus donnert « oder is dies illuxisset (Cic. Div. 1, 50) im Lateinischen relativ selten sind und deswegen bereits von Priscian als künstliche Ausdrücke der Prosopopöie betrachtet wurden (cf. Prisc. constr. Constr. 17.117ff.), sind sie in anderen indogermanischen Sprachen, vor allem im Indoiranischen und Griechischen, weitaus verbreiteter. Dabei wird das Agens entweder als Gottheit, die als Urheber der meteorologischen Phänomene betrachtet wurde, oder als Element der Natur (Tag, Himmel, Erde, etc.) beschrieben (cf. Viti 2014, 182), wie sich aus den folgenden Beispielen aus dem Altgriechischen, Sanskrit, Altkirchenslawischen und

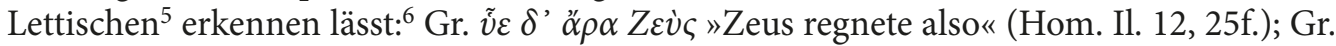
$Z \varepsilon \dot{v} \varsigma \delta^{\prime}$ 'ä $\mu v \delta \iota \varsigma \beta \rho o ́ v \tau \eta \sigma \varepsilon$ »Zeus donnerte nun « (Hom. Od. 12, 415); Skr. vato vati »Der Wind weht«; Aksl. oblakъ dъžditъ »Die Wolke regnet«; Lit. leetus līst »Der Regen regnet«; snigs snig »Der Schnee schneit«. Im Lateinischen findet sich dagegen eher, wenn auch selten, die Verbindung eines Witterungsverbums mit einem obliquen Kasus - meist dem Ablativ durch den das Agens der Verbalhandlung genauer spezifiziert wird (cf. Bauer 2000, 101): imbre lapidat »es regnet Regen«, sanguine pluit »es regnet Blut«, lapidibus pluit »es regnet Steine«. Im klassischen Latein wurden diese Strukturen, da sie gegen die herkömmlichen Kasusregeln und die Kongruenz verstiessen, normalisiert, und zwar dadurch, dass das explizite Argument als ablativus absolutus dargestellt wurde. So schreibt Cicero in Cic. Div. 2,42,14: itaque in nostris commentariis scriptum habemus: Iove tonante, fulgurante comitia populi habere nefas. "References to both types of ssubjects<, names of gods and common nouns, can also be found in expressions that are almost formulaic, such as ablative absolutes with a present participle, in which the noun refers to the underlying subject:« (Bauer 2000, 105). Dabei wurden neben Namen von Göttern, häufig die Nomen dies oder caelum genutzt, um als Argument bei einem Witterungsverb zu fungieren, z. B. fulgente caelo, lucente die oder vesperascente caelo. Daneben findet sich vor allem im Altlateinischen die Verbindung eines Witterungsverbums mit einem pronominalen Subjekt, so z. B. in der Sprache des Plautus oder des Terenz, beispielsweise hoc lucescit (Plaut. Amph. 543). In der Volkssprache und der Sprache der Dichtung werden Witterungsverben teilweise sogar transitiv gebraucht (cf. Leumann/Hoffmann/Szantyr 1997, 416), so beispielsweise ninguntque rosarum floribus umbrantes matrem comitumque catervam (Lucr. 2, 237f.). ${ }^{7}$

4 Die angeführten Beispiele entstammen Brugmann 1904, 625 und Bauer 2000, $96 \mathrm{ff}$.

5 »Expressions $\langle\ldots\rangle$ which include a noun and a verb both referring to the meteorological events, are limited basically to Balto-Slavic, although occasional instances can also be observed in other Indo-European languages« (Bauer 2000, 105).

6 Die angeführten Beispiele entstammen Viti 2014, 182 und Bauer 2000, 104f.

7 Allerdings handelt es sich hier um einen metaphorischen Gebrauch des Verbs ninguit. Bei transitivem Gebrauch der Witterungsverben wird des Weiteren meist nicht das Simplex, sondern ein präfigiertes Verbum verwendet, so z. B. hac atque illac perfluo (Ter. Eun. 105). 
Die Koexistenz von persönlichen und unpersönlichen Strukturen mit Witterungsverben im Indogermanischen warf schon früh in der Forschung die Frage auf, welche der beiden Strukturen die ursprüngliche sei. ${ }^{8}$ Während Leumann/Hoffmann/Szantyr (1997, 415) aufgrund der persönlichen Ausdrucksformen des Griechischen und Indischen noch davon ausgehen, dass »die sog. Witterungsimpersonalien (meterologische Verba) nicht idg. Alters zu sein " scheinen, und die Junggrammatiker die unpersönlich gebrauchten Witterungsverben sogar als Zeichen eines wachsenden Laizismus interpretieren, wird in der neueren Forschung davon ausgegangen, dass die Koexistenz von persönlichen und unpersönlichen Strukturen mit Witterungsverben darauf beruht, dass versucht wurde, die synchron anomalen Witterungsverben der normalen Argumentenstrukturen mit einem expliziten durch den Nominativ markierten Subjekt anzupassen. Viti $(2014,183)$ vergleicht den Gebrauch von Göttern und Naturgewalten als Agens von Witterungsverben mit dem Gebrauch eines leeren Subjekts (dummy subject), welches kein Argument darstellt, da es keine semantische Rolle innehat, in den modernen indogermanischen Sprachen, z. B.Dt. es regnet oder Engl. it rains, da diese Subjekte ebenfalls nicht von einem Adjektiv oder Genitiv modifiziert würden. Sie folgert daher, «dass, wenn eine Sprache einen Wandel im Ausdruck der grammatischen Person oder des Subjekts im Bereich der Witterungsverben hat, ein solcher Wandel von unpersönlichen zu persönlichen Strukturen oder vom impliziten zum expliziten Subjekt verläuft und nicht umgekehrt (Viti 2014, 184).

Im Gegensatz zu den indogermanischen Sprachen weist das biblische Hebräische neben den unpersönlichen Strukturen vermehrt einen persönlichen Gebrauch der Witterungsverben auf, ${ }^{9}$ bei welchem meist Gott als Agens fungiert, und das Verb selbst sowohl transitiv als auch intransitiv gebraucht werden kann. So finden sich im Hebräischen beispielsweise folgende Witterungsverben ${ }^{10}$ : regnen (lassen) « $m t r$ im Hifil ${ }^{11},{ }^{12}$ (cf.

${ }^{8}$ Cf. z. B. Brugmann (1904, 625f.): „Ob der subjektische Typus oder subjektlose der ursprünglichere gewesen ist, ist nicht zu entscheiden; das Vorhandensein der Personalendung beweist nichts für die Urspünglichkeit des subjektischen Typus. Eventuell sind also die als Impersonale bezeichneten eingliedrigen Äusserungen, wenn auch schon in uridg. Zeit, aus zweigliedrigen hervorgegangen.«.

9 Im modernen Hebräischen überwiegen dagegen unpersönliche Konstruktionen mit »circumstantial predicates« (cf. Berman 1997, 324), beispielsweise קר שם (kalt.3.ms dort)»es ist kalt dort« (cf. Melnik 2013).

${ }^{10}$ Die Angabe hebräischer Verben erfolgt stets in ihrer Wurzelstruktur.

11 »Zur Modifikation der Bedeutung einer Verbalwurzel kennen die semitischen Sprachen eine Reihe besonderer Verbalstammbildungen, die durch präfigierte oder infigierte Konsonanten oder durch die Längung des mittleren Konsonanten oder Vokals gebildet werden « (Stempel 1999, 110). Traditionell werden im Hebräisch sieben verschiedenen Stammbildungen unterschieden, und zwar der Grundstamm Qal oder Paal sowie sechs davon abgeleitete Stammbildungen (Nifal, Piel, Pual, Hitpael, Hifil und Hofal), die verschiedene semantische Beziehungen zum Qal aufweisen, z. B. Passivierung oder Kausativierung (cf. Merwe, Naudé \& Kroeze 1999, 73). Der Grundstamm Qal oder Paal gebraucht die unveränderte Verbalwurzel. »The Qal stem formation has no specific meaning per se« (Merwe, Naudé \& Kroeze 1999, 76). Dennoch kann innerhalb des Grundstammes zwischen Aktionsverben, die eine Handlung ausdrücken, und stativen Verben, die einen Zustand beschreiben, unterschieden werden. Das Nifal stellt den N-Stamm des Hebräischen dar, d. h. diejenige Stammbildung, welche ein präfigiertes $n$ aufweist (cf. Jenni 1997, Siebesma 1990). Zunächst kann das Nifal als Reflexiv zum Qal betrachtet werden; häufig besitzet es jedoch auch eine tolerative, reziproke oder mediale Bedeutung (cf. Lettinga 1991, 85f.). Ein Grossteil der Nifal-Bildungen kann auch als Passivierung des Qals aufgefasst werden. Piel und Pual stellen die D-Stämme des Hebräischen dar, d. h. diejenigen Verbalbildungen, die sich durch die Verdoppelung des mittleren Radikals kennzeichnen. Das Piel kann grundsätzlich - wie der semitische D-Stamm im Allgemeinen - als Intensivbildung des Qal aufgefasst werden (cf. Jenni 1968, Jenni 2000), weist im Hebräischen jedoch weitaus häufiger eine faktitive Bedeutung auf: »The Piel indicates the cause that places an object in the condition to which the Qal form (with a stative meaning) of the same stem refers (Merwe, Naudé \& Kroeze 1999, 80). Des Weiteren wird häufig eine resultative Bedeutung mithilfe des Piels ausgedrückt: »The Qal may describe the verb as a process that occurs, 


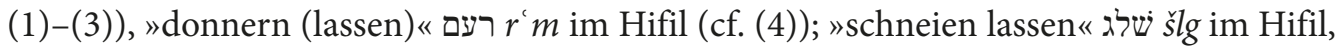
»blitzen« ברק brq im Qal (cf. (5)):

(1) Gen 2,5:

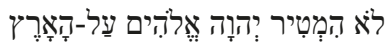

JHWH, der Herr, liess es nicht auf die Erde regnen.

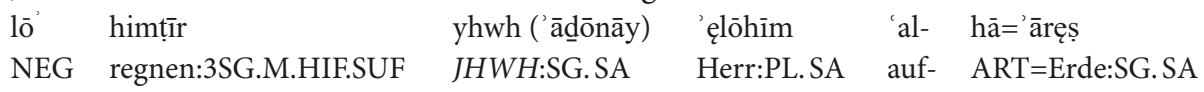

(2) Gen 19,24

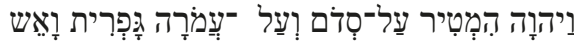

Und JHWH liess auf Sodom und Gamorra Schwefel und Feuer regnen.

$\mathrm{w}^{\mathrm{e}}=$ yhwh ('ādōnāy) hịimtị̂r 'al- $\quad$ sedōm

und=JHWH:SG.SA regnen:3SG.HIF.SUF auf- Sedom:SG.SA

$\mathrm{w}^{\mathrm{e}}=$ 'al- $\quad$ 'amōrā $\quad$ goprīit $\quad \mathrm{w}^{\mathrm{e}}=$ 'ēš

und=auf- Gamorra:SG.SA Schwefel:SG.SA und=Feuer:SG.SA

(3) $\operatorname{Ex} 9,23$

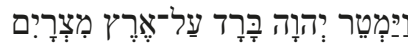

Und JHWH liess Hagel auf das Land Ägypten regnen.

$\begin{array}{lllll}\text { wayyamtẹer } & & \text { yhwh ('ādōnāy) } & \text { bārād } & \text { 'al- } \\ \text { regnen:3.SG. M. HIF. WC.PRÄ } & \text { JHWH:SG.SA } & \text { Hagel:SG.SA } & \text { auf- } \\ \text { 'ęręṣ } & \text { mișrāîm } & & & \\ \text { Land:SG. SC } & \text { Ägypten:SG.SA } & & \end{array}$

(4) $1 . \operatorname{Sam} 7,10$

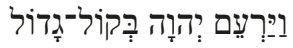

Und JHWH donnerte mit lauter Stimme.

$\begin{array}{llll}\text { wayyar'ēm } & \text { yhwh ('ādōnāy) } & \mathrm{b}^{\mathrm{e}}=\mathrm{q} o ̄ l & \text { gādōl } \\ \text { donnern:3.SG. M.HIF. WC.PRÄ. } & \text { JHWH:SG.SA } & \text { mit=Stimme:SG.SA } & \text { gross:SG.SA }\end{array}$

(5) Ps 144,6

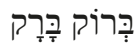

Sende Blitze! ${ }^{13}$

b rōq bārāq

blitzen:IMP.M.SG. QAL Blitz:SG.SA

while the Piel describes the result of that process« (Merwe, Naudé \& Kroeze 1999, 80). Vereinzelt kann das Piel auch mit einer deklarativen Bedeutung verbunden sein; Denominativa im Piel sind hingegen oft mit einem privativen Sinn verknüpft (cf. Lettinga 1991, 86). Der zweite Dopplungsstamm des Hebräischen, das Pual, bildet das Passiv zum Piel. Das Hitpael stellt den Dt-Stamm, d. h. den Dopplungsstamm mit präfigiertem $t$, des Hebräischen dar. Grundsätzlich kann das Hitpael als Reflexiv des Piels, seltener auch als Reflexiv des Qals, betrachtet werden. Mitunter drückt das Hitpael auch eine reziproke Handlung aus. Vereinzelt ist das Hitpael auch mit einer medialen, imitativen oder passiven Bedeutung verbunden (cf. Lettinga 1991, 86f.). Hifil und Hofal sind die H-Stämme des Hebräischen. Das Hifil bildet den Kausativ zu einem transitiven oder intransitiven Qal, vereinzelt ist das Hifil auch mit einer deklarativen Bedeutung verbunden. Das Hofal bildet schliesslich das Passiv des Hifils.

12 Selten auch גשו im Hifil (cf. Jer 14,22).

${ }^{13}$ Im Hebräischen findet sich hier eine figura etymologica mittels der Wurzel ברק, die sich aus dem Imperativ Sg. m. Qal sowie der dazugehörigen Nominalbildung zusammensetzt. Daher wäre die wörtliche Übersetzung: »Blitze Blitze!«. 
Auffällig ist der vorherrschende Gebrauch der Witterungsverben im Hifil, ${ }^{14}$ d. h. im Kausativstamm. Dadurch wird zwar Gott als Agens gekennzeichnet, dessen Handlung jedoch darin besteht, ein zweites Agens zur eigentlichen Handlung, d. h. dem Witterungsgeschehen zu veranlassen. Dieses zweite Agens bleibt dabei implizit und wird nicht genannt. Durch die Wahl des Hifils wurde somit ursprünglich betont, dass keine wirklichen belebten Partizipanten an der Handlung beteiligt sind, sondern spontane Prozesse der Natur beschrieben werden. Diachron wurde die kausative Konnotation des Hifils bei den Witterungsverben dann opak und grammatikalisiert, was einerseits dadurch verdeutlicht wird, dass bei vielen Witterungsverben nur das Hifil belegt ist, andererseits jedoch auch durch die Tatsache, dass die Witterungsverben selbst bei unpersönlichem Gebrauch das Hifil beibehalten (cf. (6)): ${ }^{15}$

(6) Ps 68,15

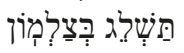

Es schneit auf dem Zalmon.

tašlēg

regnen:3.SG.F. HIF.PRÄ

$\mathrm{b}^{\mathrm{e}}=$ șalmōn

auf=Zalmon:SG.SA

Häufig wird das Witterungsgeschehen im biblischen Hebräischen auch in eine transitive Struktur mit dem Verb נתן "geben" eingebunden, wobei wiederum Gott als Agens fungiert und das Nomen des Witterungsgeschehens selbst als Patiens (cf. (7)-(9)):

(7) Ps 147,16

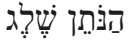

der, der Schnee gibt

ha=nōtēn

šęlęg

ART=geben:PTCP.SG.M

Schnee:SG.SA

(8) Job 5,10

הַּוֹתָן מָטָר

der, der Regen gibt

ha=nōtēn

mātār

ART=geben:PTCP.SG.M

Regen:SG.SA

Eine weitere Möglichkeit, das Witterungsgeschehen im biblischen Hebräisch auszudrücken, besteht darin, eine Verbindung aus dem Verb היה מsein" und einem Witterungsnomen zu bilden (cf. (9)) oder das Witterungsgeschehen in einem Nominalsatz zu beschreiben (cf. (10)):

${ }^{14}$ Die Wurzel ברק ״blitzen«, welche im angeführten Beispiel im Qal auftritt, weist in der gesamten hebräischen Bibel nur eine Belegstelle (Ps 144, 6) auf. Die ausserbiblischen Quellen verwenden die Wurzel stets im Hifil. Auch im modernen Hebräischen wird das Hifil verwendet.

15 Die Tatsache, dass sich die meisten unpersönlich gebrauchten althebräischen Witterungsverben des Hifils bedienen, ist auch vor dem Hintergrund interessant, dass die übrigen Impersonalia des Biblischen Hebräischen fast ausschliesslich im Qal belegt sind (cf. Waltke/O'Conner 1990, 376). Auch dies ist ein Beleg für das Verblassen der ursprünglich kausativen Konnotation des Hifils. Bei unpersönlichem Gebrauch wird die 3. Person Femininum als default-Form gebraucht. 
(9) Dt $11,17^{16}$

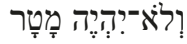

und es wird Regen haben

$\begin{array}{lll}\mathrm{w}^{\mathrm{e}}=\mathrm{lo}^{\prime} & \text {-yihyę } & \text { mātāār } \\ \text { und=NEG } & \text { sein:3.SG.M.QAL.PRÄ } & \text { Regen:SG.SA }\end{array}$

(10) 1. Sam 29,10

וְאוֹר דֶכֶם וְלְכוּ

Wenn es (genug) Licht für Euch (hat), geht!

$\mathrm{w}^{\mathrm{e}}=$ 'ōr lākęm $\quad \mathrm{w}^{\mathrm{e}}=$ lēknū

und=Licht:SG.SA für=2.PL.POSS. und=gehen:IMP.M.PL.QAL

Diese Ausdrucksweise kommt dem unpersönlichen Gebrauch der Witterungsverben im Indogermanischen am nächsten. Betrachtet man nun den Gebrauch der Witterungsverben bzw. die sprachliche Beschreibung des Witterungsgeschehens im biblischen Latein, so fällt zunächst auf, dass der persönliche Gebrauch der Witterungsverben überwiegt wie von García de la Fuente (1990, 271f.) am Beispiel von pluit konstatiert wird: »Empleo personal de pluere en contra de la norma clásica. « Dabei ist jedoch zu beachten, dass die drei häufigsten im Hebräischen identifizierten Strukturen zum Ausdruck des Witterungsgeschehens auch verschiedenartig ins Lateinische der Vulgata übertragen werden (cf. (11)-(16)). Der persönliche Gebrauch der Witterungsverben mit Gott als Agens wird in dieser Weise im Lateinischen wiedergegeben, wobei jedoch die Kausativität des Hifils nicht übertragen wird. ${ }^{17}$ Auch der teilweise transitive Gebrauch der Witterungsverben ist im biblischen Latein verbreitet.

(11) non enim pluerat Dominus Deus super terram

Gen 2,5

(12) igitur Dominus pluit super Sodomam et Gomorrham sulphur et ignem

Gen 19,24

(13) pluitque Dominus grandinem super terram Aegypti

Ex 9,23

(14) intonuit autem Dominus fragore magno

1.Sam 7,10

(15) fulgura coruscationem

Ps 144,6

(16) nive dealbabuntur in Selmon

Ps 68,15

Der persönliche Gebrauch der Witterungsverben wird somit bei expliziter Nennung Gottes als Agens vom biblischen Hebräischen ins Lateinische der Vulgata übertragen (cf. (11)-(13)). Wird hingegen kein göttliches Agens im Hebräischen verwendet wie dies in Ps 68, 15 der Fall ist (cf. (6)), so wählt Hieronymus für das Lateinische eine passive Konstruktion ohne explizites Agens, wobei das Witterungsgeschehen ebenfalls als Patiens durch den obliquen Kasus gekennzeichnet wird (cf. (16)).

16 Beispielsweise auch 1 Kö 8,35 und 2 Chr 7,13.

17 Synchron dürfte der ursprünglich mithilfe des Hifils ausgedrückte kausative Gebrauch der Witterungsverben bereits durch Grammatikalisierung so opak gewesen sein, dass er für die Sprecher des Biblischen Hebräischen nicht mehr erkennbar war. Darauf deutet auch hin, dass die Witterungsverben meist nicht im Qal belegt sind. 
Die Struktur נתן "geben" + zugehöriges Witterungsnomen wird meist als calque verbum pro verbo ins Lateinische übertragen (cf. (17)-(18)); teilweise wird die Struktur jedoch durch Passivierung enttransitivisiert und so dem Lateinischen unpersönlichen Gebrauch der Witterungsverben angenährt, indem das Agens in den Hintergrund rückt.

(17) qui dat nivem

Ps 147,16

(18) qui dat pluviam

Job 5,10

Die Beschreibung des Witterungsgeschehens mittels des Verbs היה ״sein « und eines Witterungsnomens wird hingegen auf zwei verschiedene Arten ins Lateinische übertragen:

(19) et pluviae non descendant

Dt 11,17

(20) et non pluerit

1. Kö 8,35

(21) et pluvia non fluxerit

2. Chr. 7,13

Einerseits wird versucht, den nominalen Charakter der Witterungsbeschreibung beizubehalten, indem das hebräische Nomen übersetzt wird (cf. (19).(21)), und zwar teilweise im Singular (cf. (19)), teilweise im Plural (cf. (21)). Andererseits wird der Unpersönlichkeit der Struktur Rechnung getragen, indem sie als Impersonal, beispielweise in 1. Kö 8,35, ins Lateinische übertragen wird (cf. (20)).

Im Vergleich zum klassischen Latein weist das biblische Latein tendenziell einen Wandel von nicht-kanonisch zu kanonisch markierten Witterungsverben auf, d. h. einen Wandel von unpersönlichen zu persönlichen Strukturen. Dieser Wandel ist jedoch weniger als syntaktischer Hebraismus zu erklären als viel mehr Ausdruck der synchronen innerlateinischen bzw. innerindogermanischen Varianz, die durch den Sprachkontakt mit dem Hebräischen, in welchem diese Strukturen regelmässig grammatikalisiert sind, vermehrt zum Ausdruck kommt.

\section{Empfindungsverben}

Die Empfindungsverben als weitere Gruppe von Impersonalia im Indogermanischen wurden bereits von Brugmann (1904, 629) identifiziert, welcher sie als »Verba, die eine Affektion des Leibes oder der Seele bezeichnen ", beschrieb. Das Experiens der Empfindungsverben ist meist belebt, während der Stimulus unbelebt ist, sodass die Struktur dieser Prädikate oft als invertiert bezeichnet wird: "Dans ce champ, les verbes de perception forment un groupe à part bien délimité, avec une spécificité sémantique nettement perceptible : avec ces verbes-là, il n'y a pas d'action qui parte d'un actant animé; c'est plutôt l'inverse puisque c'est lui qui subit l'expérience. Le vecteur sémantique verbal est interverti : au lieu dêtre le point de départ du processus décrit par le verbe, l'actant animé devient le point d'arrivée. Dans le cadre général de la bivalence, les verbes de perception posent donc un problème de nature sémantique." (Bossong 1997, 259).

Das Lateinische weist beispielsweise folgende unpersönlich gebrauchte Empfindungsverben auf, bei welchen das Experiens mit dem Akkusativ und der Stimulus mit einem weiteren obliquen Kasus ${ }^{18}$ oder einer Infinitivkonstruktion ${ }^{19}$ markiert

18 Z.B. me paenitet eius.

19 Z. B. Non libet omnia criminari. (Cic. Ver. 2,4,59) 
wird: ${ }^{20}$ me miseret »ich habe Mitleid«, me paenitet «ich bereue«, me piget »es tut mir Leid«, me pudet »ich schäme mich « und me taedet »mich ekelt es «. ${ }^{21}$ Bei placet "gefallen" steht das Experiens hingegen im Dativ. Auch in anderen alten indogermanischen Sprachen weisen Empfindungsverben häufig eine nicht-kanonische Struktur auf, z. B.Skr. ná mā sramat "nicht ergreife mich Ermüdung«; Got. huggreip mik "mich hungert«, paúrseip mik »mich dürstet«; Lit. mán sálta »mich friert«, man nórisi «ich habe Lust «. ${ }^{22}$ Dennoch überwiegt bei lateinischen Empfindungsverben eine kanonische Markierung: »Bien que l'emploi impersonnel se soit sur certains points maintenu ou même développé, il y avait une forte tendance contraire vers la tournure personnelle. En particulier, les impersonnels de sentiment paraissaient isolés au milieu de verbes de sens voisin, mais tous personnels" (Ernout/Meillet 1953, 210f.). Zu diesen Verben, bei welchen das Experiens mit dem Nominativ markiert wird, gehören z. B. algeo »mir ist es kalt«, esurio »ich habe Hunger", sitio »ich habe Durst «, gaudeo »ich freue mich «, laetor »ich freue mich «, memini »ich erinnere mich «, reminiscor »ich erinnere mich «, obliviscor »ich erinnere mich « und video »ich sehe«. Synchron findet sich im Lateinischen häufig eine Variation zwischen kanonischen und nicht-kanonischen Strukturen, z. B. me miseret vs. misereor. So findet sich im Altlateinischen im Gegensatz zum klassischen Latein, wo die Verben timeo, metuo und vereor eine kanonische Struktur nach sich ziehen, auch die unpersönliche Form mihi veretur »ich fürchte mich $\aleph^{23}$. Neben der synchronen Varianz ${ }^{24}$ zwischen persönlichen und unpersönlichen Strukturen treten im Lateinischen zusätzlich »einige von Deponentien gebildete Impersonlien auf wie altlat. me miseretur (Leumann/Hoffmann/Szantyr 1953, 416).

Diachron weisen die Empfindungsverben eine Entwicklung von nicht-kanonischen Strukturen zu kanonischen Strukturen auf: »There is a well-established diachronic tendency for oblique experiencer arguments to acquire behavioral subject properties" (Haspelmath 2001, 75). Bei den häufig gebrauchten Empfindungsprädikaten (cf. Menge 2009, 395ff.) interest und refert »es ist von Bedeutung, Wert für jemand, jemand hat Interesse an etwas, es kommt darauf an« bleibt die nicht-kanonische Markierung mit obliquem Experiens regelmässig auch im klassischen Latein«. Dabei wird das Experiens entweder mit dem Genitiv ${ }^{25}$ (im Fall eines Nomens) oder mit der femininen Form eines Pronomens ${ }^{26}$ markiert.

Im biblischen Hebräischen bilden die Empfindungsverben wie im Semitischen im Allgemeinen die grösste Gruppe der Impersonalia. Syntaktisch wird hier das Experiens

${ }^{20}$ Die Aufzählung beruht auf Ernout/Meillet 1953, 209 und Menge 2009, 309. Auch andere alte indogermanische Sprachen gebrauchen bei den nicht-kanonischen Strukturen der impersonalen Empfindungsverben häufig den Akkusativ oder Dativ zum Ausdruck des Experiens und einen anderen obliquen Kasus zur Markierung des Stimulus, z. B. altisländisch mik iðrar pess »ich (AKK) bereue es (GEN)«.

${ }^{21}$ Teilweise weisen die Verben auch im Perfekt nicht-kanonische Markierung auf: »Trois de ces verbes ont, en outre, conservé un perfectum impersonnel: (me) misertum est pour miseret; (me) puditum est pour pudet; (me) pertaesum est pour taedet; à côté de (me) puditum est apparaît d'ailleurs (me) puduit « (Ernout/ Meillet 1953, 209).

22 Die Beispiele beruhen auf Brugmann 1904, 629 und Viti 2014, 156.

${ }^{23}$ Z.B. si tui veretur te progenitoris (Accius fr. 76).

${ }^{24}$ Diese synchrone Varianz zeigt sich bereits im Altlateinischen, beispielsweise für pudet, welches in Pl. Cas. 877f. innerhalb eines Satzes sowohl persönlich als auch unpersönlich gebraucht wird: Ita nunc pudeo atque ita nunc paueo atque ita inridiculo sumus ambo. Sed ego insipiens noua nunc facio: pudet quem prius non puditumst umquam.

25 Z.B. Petri interest »es ist für Peter wichtig«.

${ }^{26}$ Z. B. mea interest »es ist für mich wichtig". 
meist in einer Präpositionalphrase in -ל an das unpersönliche Verb, welches die default form 3. Sg aufweist, angeschlossen wie aus den folgenden Beispielen (22) bis (26) ersichtlich ist: ${ }^{27}$

(22) Jon 4,1

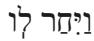

Es erzürnte ihn.

$\begin{array}{ll}\text { wa=ȳihar } & \mathrm{l}=\overline{\mathrm{o}} \\ \text { und=erzürnen:3.SG.M. } & \text { für=3.SG.M. }\end{array}$

(23) 1. Sam. 16,23

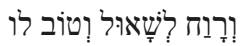

Schaul fühlte sich erleichtert und gut. ${ }^{28}$

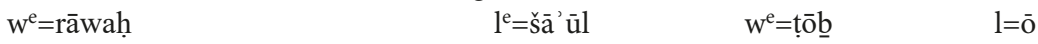

und=erleichtern:3.SG.M. für=Schaul und=gut:ADJ für=3.SG.M.

(24) Job 20,22

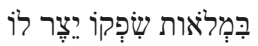

In der Fülle seines Überflusses war es ihm Angst.

bi=mel'ōt śipqō yēșerr $\quad l=\bar{o}$

in=Fülle:SG.SC Überfluss:SG.3.SG.POSS Angst haben:3.SG.M.QAL.PR für=3.SG.M.

(25) Job 3,13

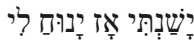

Ich würde dann schlafen und mir wäre es ruhig.

$\begin{array}{llll}\text { yāšantī } & \text { 'āz } & \text { yānūăh } & l=\overline{1} \\ \text { schlafen:1.SG.QAL.SUFF } & \text { dann } & \text { ruhig sein:3.SG.M.QAL.SUFF } & \text { für=1.SG }\end{array}$

(26) Prov 24,25

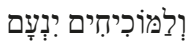

Und denen, die Recht sprechen, ist wohl.

$\mathrm{w}^{\mathrm{e}}=\mathrm{la}=\mathrm{mō} \underline{\underline{1}} \mathbf{i} h \bar{i} \mathrm{~m}$

yin'ām

und=für=Recht sprechen:PART.PL.HIFIL.SA

wohl sein:3.SG.M.QAL.PRÄ

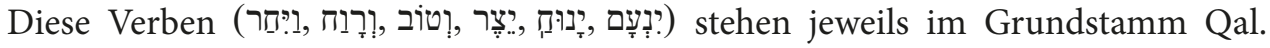
Betrachtet man die lateinische Übersetzung dieser Verse, so fällt auf, dass sämtliche Prädikate kanonisch markiert sind und eine persönliche Konstruktion nach sich ziehen (cf. $(27)^{29}-(31)$ ):

(27) et iratus est

(28) et refocillabatur Saul, et levius habebat

(29) omnis dolor irruet super eum.

(30) somno meo requiescerem

(31) qui arguunt eum laudabuntur
Jon 4,1

1. Sam 16,23

Job 20,22

Job 3,13

Prov 24,25

27 Die Beispiele entstammen Waltke/O’Connor 1990, 377.

28 Wörtlich: »Es war weit für Schaul und es war gut für ihn«.

29 Die lateinsche Übertragung iratus est wählt zwar eine kanonisch markierte Struktur, bei welcher das Experiens mithilfe des Nominativs kodiert wird, zeigt aber die geringe Transitivität durch die Verwendung des Deponens irari an. 
Dies hat zur Folge, dass das Experiens, das im Hebräischen einen obliquen Kasus aufweist und mittels einer Präpositionalphrase ausgedrückt wird, im Lateinischen meist zum grammatikalischen Subjekt wird, welches mit dem Nominativ kodiert ist. Eine weitere Möglichkeit der Übersetzung besteht darin, den Stimulus, welcher im hebräischen impersonalen Verb implizit enthalten ist, zum grammatikalischen Subjekt des lateinischen Satzes zu machen und den Experiens entweder in einem obliquen Kasus oder mithilfe einer Präpositionalphrase anzuschließen.

\section{Modalverben}

Eine weitere Gruppe der Impersonalia innerhalb der Indogermania stellen die sogenannten Modalverben dar, welche modale Urteile oder Aussagen über die Notwendigkeit, Möglichkeit oder Geeignetheit bezeichnen (cf. Viti 2014, 157f.), z. B. Lat.

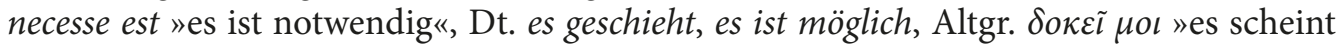
mir«, $\delta \eta \lambda o \tilde{~} \mu o \iota$ »es ist mir klar« oder Lit. mán reîkia »ich muss«, mán rupi »ich sorge«. Dabei lassen sich nach Lambert $(1997,301)$ zwei Typen von Modalverben unterscheiden, und zwar lexikalische Impersonalia (»impersonnel lexical«), z. B. decet »es ziemt sich«, necesse est "es ist notwendig", licet "es ist erlaubt " oder oportet »es gebührt " und morphologische Impersonalia ${ }^{30}$ (»verbe impersonnalisé par construction«), z. B. iuvat »es freut «, constat »es ist bekannt", praestat »es ist besser», apparet »es ist offenbar», liquet »es ist klar«, evenit »es ereignet sich«, accidit »es ereignet sich«, contigit »es ereignet sich«, fallit »es entgeht «, fugit »es entgeht«, praeterit »es entgeht «. Im Gegensatz zu den meisten anderen Impersonlia bleiben Modalverben diachron gesehen nicht nur nicht-kanonisch markiert, sondern bilden sogar neue unpersönliche Strukturen (cf. Bauer 2000, 121). ${ }^{31}$ So entwickelt beispielsweise das klassisch nur persönlich gebrauchte debeo »ich muss « als debet + Infinitiv "man muss « eine unpersönliche Konstruktion, ${ }^{32}$ ähnlich entwickelt auch possum »ich kann« in potest + Infinitiv »es ist möglich« eine unpersönliche Konstruktion (cf. Viti 2014, 185f.).

Im biblischen Latein sind impersonale Modalverben hingegen sehr spärlich vertreten und meist auf das Neue Testament beschränkt, ${ }^{33}$ während sie im alttestamentarischen Teil der Vulgata praktisch nicht vertreten sind. Dieser Mangel an Modalverben im biblischen Latein des Alten Testaments lässt sich höchst wahrscheinlich damit erklären, dass »a dearth of modal auxiliaries causes Biblical Hebrew to employ a relatively simprovished coding

${ }^{30}$ Morphologische Impersonalia werden von Verben gebildet, die je nach Kontext auch einen persönlichen Gebrauch aufweisen können, cf. Lambert (1997, 301): «ont deux emplois, personnels et impersonnels; le second emploi est déterminé par la construction syntaxique.«

31 Dies lässt sich gut in der Entwicklung des Englischen erkennen, welches, obwohl es sehr arm an unpersönlichen Konstruktionen ist, diese bei den Modalverben bewahrt hat, z. B. it seems, it appears, it occurs, it behaves, it fits (cf. Viti 2014, 185). Viti $(2014,186)$ sieht den Grund dieser Entwicklung, die entgegen dem allgemeinen drift der Impersonalia hin zu persönlichen Konstruktionen verläuft, darin, »dass Modalverben einen breiteren syntaktischen Umfang (scope) « als andere Impersonlia haben.

32 Z. B. Mul. Chir. 33: sanguinem emittere [.] de capite debet "man soll Blut von dem Kopf freilassen" (Viti 2014, 186).

${ }^{33}$ Hier zeigt sich deutlich das Wesen des biblischen Lateins als Übersetzungssprache. Da das im Koine-Griechischen, d. h. in einer indogermanischen Sprache verfasste Neue Testament bereits in der Ausgangssprache Modalverben aufweist, welche unpersönliche Konstruktionen nach sich ziehen, werden diese ebenfalls in der Zielsprache, d. h. im Lateinischen der Vulgata verwendet. Im alttestamentarischen Teil der Vulgata wird hingegen versucht, die hebräischen Konstruktionen nachzuahmen. 
system` for modality « (Callaham 2010, 36) stattfand. Aussagen über Notwendigkeit, Möglichkeit, Gelegenheit, etc. werden im biblischen Hebräischen entweder mithilfe morphologischer Verfahren ${ }^{34}$, modaler Adverbien oder mittels persönlich konstruierter Modalverben ${ }^{35}$ formuliert.

\section{Possessive Strukturen}

Eine weitere Gruppe von Prädikaten, welche sprachübergreifend relativ häufig eine nicht-kanonische Markierung aufweisen können, bilden Verben des Besitzens, des Vorhandenseins sowie des Mangels: "The predicates of this class express possession, (non-)existence and lacking, and being (in-)sufficient " (Oinishi 2001, 34). Nichtkanonische Strukturen zur Bezeichnung des Besitzes können für das Urindogermanische rekonstruiert werden ${ }^{36}$ und sind in den alten urindogermanischen Sprachen belegt: Dabei wird "eine nicht-kanonisch markierte Konstruktion mit dem Verb ssein<, in der das (normalerweise unbelebte) Besessene im Nominativ steht, während der menschliche Besitzer durch einen obliquen Nominal ausgedrückt wird « (Viti 2014, 158f.) verstanden. Während im lateinischen mihi est aliquid der Besitz mithilfe des Dativs markiert wird und der Dativ auch im Hethitischen ${ }^{37}$ als obliquer Kasus zur Besitzmarkierung herangezogen wird, erfolgt die Markierung im Altindischen ${ }^{38}$ mit dem Genitiv, im Altkirchenslawischen mit einem lokativischen Präpositionalausdruck. ${ }^{39}$

Während im frühen und klassischen Latein die mihi est aliquid -Strukturen weit verbreitet sind und auch mit negativer Polarität auftreten können (mihi deest aliquid), kann ein diachroner Verfall dieser Struktur konstatiert werden: "While in Early Latin sthe dative of possession is a very common idiom ` (Bennett 1914, 159), the use of mihi est constructions in the later periods became increasingly confined to a specific group of nouns. The gradual restriction of the noun is integrated in the developement that eventually led to the replacement of mihi est by habeo (Bauer 2000, 181).

Auch in den semitischen Sprachen wird häufig eine nicht-kanonische Struktur zum Ausdruck des Besitzes verwendet. Im Hebräischen wird dabei der Besitzer meist in einer Präpostionalphrase mit $-l^{e}$ angeschlossen. Die Prädikation erfolgt entweder durch eine von der Wurzel היה haya ${ }^{h}$ "sein" abgeleiteten Verbalform oder durch die existenziellen

34 Aussagen, die im Lateinischen mithilfe unpersönlicher Modalverben ausgedrückt werden, entsprechen häufig einem in der hebräischen Präfixkonjugation formulierten Ausdruck.

35 Z. B. אבדָה "gewillt sein«.

${ }^{36}$ Ein Beleg hierfür ist auch die Abwesenheit einer einheitlichen Wurzel für das transitive Verb »haben « im Indogermanischen: Lat. habeo, Altgr. $\chi^{\prime} \chi \omega, \mathrm{kl}$. Arm. ownim, Lit. turèti, etc.

37 Z.B. ammuk tuppi ešzi ich: DAT Tafel: NOM sein: PRS. IND3SG

"Ich habe eine Tafel.» (cf. Viti 2014: 159)

38 Z.B. mama pustakam asti ich: GEN $\quad$ Buch(N): NOM.SG sein: PRS. IND3SG

»Ich habe ein Buch." (cf. Viti 2014: 160)

39 Hier stellt sich die Frage, welcher oblique Kasus ursprünglich zur Markierung des Besitzers diente. Nach Bauer $(2000,193)$ müsste der Dativ ursprünglich sein, da der possessive Genitiv mehr den attributiven als den prädikativen Besitz ausdrückt. Viti $(2014,160)$ hält es hingegen für plausibel, dass beide Kasus bereits im Urindogermanischen zur Markierung des Besitzes herangezogen wurden, da z. B. im Altgriechischen sowohl der Dativ als auch der Genitiv den Besitz markieren können, zumal auch in den modernen indogermanischen Sprachen aufgrund der komplexen Semantik des Besitzes (konkret vs. abstrakt, alienabel vs. inalienabl, etc.) mehrere verschiedene Strukturen zur Verfügung stehen. 
Partikel שֶי.. yēš »es gibt« oder »es gibt nicht«. Im Gegensatz zu den indogermanischen Sprachen, bei welchen der Besitz durch den Nominativ markiert wird, wird im Hebräischen der Besitz wie bei einem transitiven Verb angeschlossen, d. h. im Falle eines determinierten Besitzes mithilfe der nota accusativi אֶ eț, wie aus den Beispielen (32)(36) deutlich wird:

(32) Num 5,10

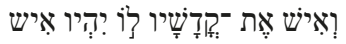

Ihm sollen die heiligen Dinge eines jeden gehören. ${ }^{40}$

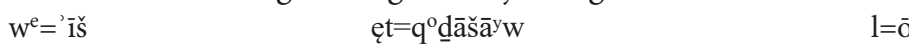

und=Mann:SG.M.SA AKK=heilig:PL.M.3.SG.M.POSS für=3.SG.M.

yihyū

sein:3.PL.Qal.PRÄ Mann:SG.M.SA

(33) Ez 35,10

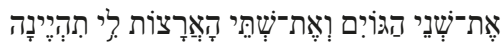

Die zwei Nationen und die zwei Länder sollen mir gehören.

\begin{tabular}{|c|c|c|}
\hline ęt=šenē & ha=gōyim & $\mathrm{w}^{\mathrm{e}}={ }^{\prime} \mathrm{et}=\breve{s}^{\mathrm{e}} \mathrm{te}$ \\
\hline AKK=zwei.M.SC & ART=Volk:PL.M & und $=\mathrm{AKK}=$ zwei:F.SC \\
\hline hā= ${ }^{a}$ rāṣōot & $\mathrm{l}=\overline{\mathrm{l}}$ & tihh ${ }^{\mathrm{e}}$ ēnā \\
\hline ART=Land:PL.F & für $=1 . S G$ & sein:3.PL.F.QAL.PRÄ \\
\hline
\end{tabular}

(34) Gen 44,20

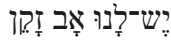

Wir haben einen alten Vater.

yęš=-lā=nū 'āb $\quad$ zāqēn

$\mathrm{COP}=$ für $=1 . P L \quad$ Vater.SG.M.SA alt:SG. M.SA

(35) Gen 43,7

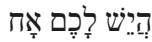

Habt ihr einen Bruder?

hayěš lā=kęm 'ạh

Q.COP für=2.PL.M. Bruder:SG.M.SA

(36) Gen 33,9

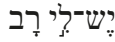

Ich habe genug.

yeš-l=ī rāb

COP-für=1.SG gross:ADJ.SA

Bei der Übertragung der unpersönlichen possessiven Strukturen des Hebräischen ins Lateinische der Vulgata werden diese - wie in den angeführten Beispielen ersichtlich ist — teilweise ebenfalls mithilfe einer unpersönlichen mihi est aliquid Struktur wiedergegeben (cf. (39)), weitaus häufiger wird jedoch eine kanonische Struktur verwendet: Syntaktisch wird dies realisiert, indem entweder der Besitz mithilfe des Nominativs als Subjekt

${ }^{40}$ Im Hebräischen stellt das vorangestellte וְ einen casus pendens dar, genauer gesagt einen nominativus pendens, d. h. einen dem eigentlichen Satzzusammenhang vorangestetellten, besonders topikalisierten Nominativ. Die Nominalform איש wurde im Hebräischen als Indefinitpronomen grammatikalisiert. 
markiert und der Besitzer in Form eines kongruenten Possessivpronomens (cf. (38)) oder eines Genitivattributes (cf. (37)) angeschlossen wird oder indem eine transitive Struktur mit dem Verb habeo gewählt wird (cf. (40), (41)). In diesem Fall wird der Besitzer mithilfe des Nominativs als syntaktisches Subjekt markiert; der Besitz wird als Akkusativobjekt angeschlossen.

(37) et quidquid in sanctuarium ipsius erit

Num 5,10

(38) duae gentes et duae terrae meae erunt

Ez 35,10

(39) est nobis pater senex

Gen 44,20

(40) si haberemus fratrem

Gen 43,7

(41) habeo plurima

Gen 33,9

Das Lateinische der Vulgata weist folglich die Tendenz auf, possessive Strukturen vermehrt kanonisch markiert auszudrücken.

\section{Unpersönliches Passiv}

Das unpersönliche Passiv gehört sprachübergreifend $\mathrm{zu}$ den häufigsten nichtkanonischen Strukturen. Im Gegensatz zu vielen anderen nicht-kanonischen Strukturen kann das unpersönliche Passiv unabhängig von den semantisch-lexikalischen Eigenschaften des Prädikats von einer Vielzahl von Verben gebildet werden und ist sowohl in den alten indogermanischen Sprachen, z. B. Lat. dicitur "man sagt « oder traditur »man überliefert«, als auch in den modernen, z. B. Dt. es wird getanzt oder It. si vende, belegt. In den alten indogermanischen Sprachen, z. B.im Lateinischen, Keltischen, Hethitischen und Tocharischen, wird es von einer medialen Form mit r-Endung ausgedrückt (cf. Viti 2014, 161). ${ }^{41}$ Auch in den semitischen Sprachen ist das unpersönliche Passiv belegt. Meist wird im Hebräischen hierzu der Nifal gebraucht (cf. Waltke/O'Connor 1990, 384f.), wie die Beispiele (42) bis (44) verdeutlichen:

(42) Gen 2,23

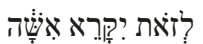

zu dieser soll Frau gesagt werden

$\begin{array}{lll}\mathrm{l}^{\mathrm{e}}=\mathrm{zō} \underline{\underline{t}} & \text { yiqqārēè } & \text { 'ššā } \\ \text { für=DEM.SG.F. } & \text { sagen:3.SG.M.NIF } & \text { Frau:SG.F.SA }\end{array}$

(43) Num 21,14

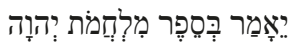

Im Buch der Kriege JHWHs wird gesagt.

$\begin{array}{llll}\text { yēāmar } & \text { b }^{\mathrm{e}} \text { sēfęr } & \text { milḥ̆mot } & \text { yhwh ('ādōnāy) } \\ \text { sagen:3.SG.M.NIF.PRÄ } & \text { in=Buch:SG.M.SC } & \text { Krieg:PL.F.SC } & \text { Gott:SG.M.SA }\end{array}$

41 „In einigen idg. Sprachen wird die Funktion des unpersönlichen Passivs durch man-Sätze ausgedrückt, die eine gewisse Konfiguratonalität voraussetzen, denn die Wörter man des Deutschen oder on des Französischen haben keine referentielle Funktion, sondern stellen eine fortgeschrittene Grammatikalisierung des Wortes `Mann` (Fr. homme) dar, das zurzeit nur ein Platzhalter ist« (Viti 2014, 162). 
(44) Is 53,5

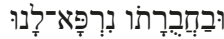

durch seine Wunden wurden wir geheilt ${ }^{42}$

$\hat{\mathrm{u}}=\underline{\mathrm{b}} \mathrm{a}=\mathrm{h}^{\mathrm{a}} \underline{\underline{b}} \mathbf{\mathrm { r }} \mathrm{a} \underline{\underline{t}} \overline{\mathrm{o}}$

nirpā' lā=nû

und $=$ durch $=$ Wunde:PL.F.POSS.3.SG.M

heilen:3.SG.M.NIF.PRÄ für=1.PL

Auch im Lateinischen der Vulgata wird das unpersönliche Passiv verwendet und zur Wiedergabe der hebräischen Konstruktion herangezogen, sofern dies wie in (45) im Lateinischen möglich ist. Kann ein lateinisches Verb, dessen hebräische Entsprechung ein unpersönliches Passiv aufweist, dieses nicht bilden, so wird in der Übersetzung eine persönliche Passivkonstruktion gewählt, wie sich in (46) und (47) erkennen lässt:

(45) dicitur in libro bellorum Domini

Num 21,14

(46) haec vocabitur Virago

Gen 2,23

(47) et livore eius sanati sumus

Is 53,5

\section{Zusammenfassung}

Die komparatistische Betrachtung (un)persönlicher Strukturen im Lateinischen der Vulgata mit den Impersonalia der alten indogermanischen Sprachen sowie den unpersönlichen Strukturen des klassischen Hebräischen zeigte einerseits deutlich den Status des Vulgata-Lateinischen als Übersetzungssprache, welche versucht, die syntaktischen Strukturen der Aussgangssprache auch in der Zielsprache abzubilden, andererseits jedoch auch, dass im Vulgata-Lateinischen hierfür keineswegs neue Syntagmata gebildet werden, sondern vielmehr auf bereits den indogermanischen Sprachen innewohnenden Konstruktionen zurückgegriffen wird. Hinsichtlich der einzelnen Gruppen der Impersonalia zeigen sich jedoch deutliche Unterschiede. Während die Witterungsverben im klassischen Lateinischen fast ausnahmslos nicht-kanonisch markiert sind, überwiegen im Lateinischen der Vulgata kanonisch markierte Witterungsverben. Dieser Wandel ist jedoch weniger als reiner syntaktischer Hebraismus zu erklären als viel mehr Ausdruck der synchronen innerlateinischen bzw. innerindogermanischen Varianz, die durch den Sprachkontakt mit dem Hebräischen, in welchem diese Strukturen regelmässig grammatikalisiert sind, vermehrt zum Ausdruck kommt. Während sowohl das klassische Lateinische wie das Indogermanische im Allgemeinen als auch das klassische Hebräische reich an unpersönlichen Empfindungsverben sind, fällt auf, dass die Übersetzung von denjenigen Prädikaten, die im Bibelhebräischen unpersönliche Empfindungsverben darstellen, zu kanonischen Strukturen im Vulgata-Lateinischen führt. Das Experiens, das im Hebräischen einen obliquen Kasus aufweist und mittels einer Präpositionalphrase ausgedrückt wird, wird im Lateinischen meist zum grammatikalischen Subjekt, welches mit dem Nominativ kodiert ist. Dieslässt sich vor allem damit erklären, dass die Lexeme, welche im Hebräischen unpersönliche Empfindungsverben sind, nicht mit den unpersönlichen Empfindungsverben des Lateinischen bzw. Indogermanischen übereinstimmen. Das Fehlen unpersönlich konstruierter Modalverben im alttestamentarischen Teil der Vulgata unterstreicht deutlich den Status des Vulgata-Lateinischen als Übersetzungssprache,

42 Wörtlich: durch seine Wunden wurde in Bezug auf uns geheilt. 
welches hier im Gegensatz $\mathrm{zu}$ den an Modalverben reichen indogermanischen Sprachen das Wesen des klassischen Hebräischen nachzuahmen versucht, welches über keine unpersönlich konstruierten Modalverben verfügt. Das unpersönliche Passiv ist schliesslich sowohl im klassischen Lateinischen als auch im klassischen Hebräischen und dem Vulgata-Lateinischen vertreten.

\section{Literaturhinweise}

Bauer B. Archaic Syntax in Indo-European: The Spread of Transitivity in Latin and French. Berlin-New York, De Gruyter, 2000.

Bennet Ch. Syntax of Early Latin. Volume 2: The Cases. Boston, Allyn and Bacon, 1914.

Berman R. A. Modern Hebrew, in: R. Hetzron (ed.) The Semitic Languages. London-New York, Routledge, 1997, 312-333.

Bossong G. Le marquage de l'expérient dans les langues d'Europe, in: J. Feuillet (éd.) Actance et valence. Berlin-New York, De Gruyter, 1997, 259-294.

Brugmann K. Kurze vergleichende Grammatik der indogermanischen Sprachen. Strassburg, Trübner, 1904.

Callaham S. N. Modality and the Biblical Hebrew Infinitive Absolute. Wiesbaden, Harrassowitz, 2010.

Ernout A. Morphologie historique du latin. Paris, Kliensieck, 1953.

García de la Fuente O. Antología del Latín Bíblico y Cristiano. Málaga, Edinford, 1990.

Haspelmath M. Non-canonical marking of core arguments in European languages, in: A. Aikhenvald et al. (eds). Non-canonical Marking of Subjects and Objects. Amsterdam-Philadelphia, John Benjamins, 2001, 53-84.

Jenni E. Das hebräische Piél. Syntaktisch-semasiologische Untersuchung einer Verbalform im Alten Testament. Zürich, EVZ-Verlag, 1968.

Jenni E. Aktionsarten und Stammformen im Althebräischen: Das Piel in verbesserter Sicht. Zeitschrift für Althebraistik 13, 2000, 67-90.

Jenni E. Nif'al und Hitpa'el im Biblisch-Hebräischen, in: E. Jenni, Studien zur Sprachwelt des Alten Testaments III. Stuttgart, Kohlhammer, 1997, 131-304.

Lambert P. L'impersonnel, in: J. Feuillet (éd.) Actance et valence. Berlin/New York, De Gruyter, 1997, 295346.

Lettinga J. P. Grammatik des biblischen Hebräisch. Basel, Immanuel, 1992.

Leumann M., Hoffmann J.B., Szantyr A. Lateinische Grammatik. Zweiter Band: Syntax und Stilistik. München, Beck, ${ }^{2} 1997$.

Leumann M., Hoffmann J. B., Szantyr A. Lateinische Grammatik. Erster Band: Lateinische Laut- und Formenlehre. München, Beck 1977.

Melnik N. Impersonal Constructions, in: G. Khan (ed.) Encylopedia of Hebrew Language, 2014. Available at: http://referenceworks.brillonline.com/entries/encyclopedia-of-hebrew-language-and-linguistics/ impersonal-constructions-EHLL_COM_00000486 (22.10.17).

Menge H. Lehrbuch der lateinischen Syntax und Semantik. Völlig neu bearbeitet von Burkhard T., Schauer M. Darmstadt, Wissenschaftliche Buchgesellschaft, ${ }^{4} 2009$.

Merwe C. H. J. van der, Naudé J. A., Kroeze J.H. A Biblical Hebrew Reference Grammar. Sheffield, SAP, 1999.

Onishi M. Introduction: Non-canonically marked subjects and objects: parameters and properties, in: A. Aikhenvald et al. Non-canonical marking of subjects and objects. Amsterdam/Philadelphia, John Benjamins, 2001, 1-52

Siebesma P. A. The Function of the Niphal in Biblical Hebrew in Relationship to Other Passive-Reflexive Verbal Stems and to the Pu'al and Hophial in Particular. Van Gorcum, Assen/Maastricht, 1990.

Stempel R. Abriss einer historischen Grammatik der semitischen Sprachen. Frankfurt am Main, Peter Lang, 1999.

Viti C. Variation und Wandel in der Syntax der alten indogermanischen Sprachen. Tübingen, Narr, 2014.

Waltke B. K., O'Conner M. An Introduction to Biblical Hebrew Syntax. Winona Lake, Eisenbrauns, 1990.

Woodcock E. C. A New Latin Syntax. Durham, Methuen, 1958.

For citation: Duerrschnabel Ch.V. (Un)Persönliche Strukturen im Lateinischen der Vulgata und ihre hebräischen Parallelen. Philologia Classica 2017, 12(2), 160-176. https://doi.org/10.21638/11701/ spbu20.2017.206 


\section{В ИВРИТЕ}

Хайа В. Дюрринабель

Феномен безличных глаголов в индоевропейских языках и, в частности, в латыни - широко дискутируемая проблема. Однако существование и функционирование безличных глаголов в латинском языке Вульгаты никогда не исследовалось с использованием сравнительного метода. Главной целью данной статьи является обсуждение использования безличных глаголов в древних индоевропейских языках и библейском иврите, а также выявление принципов, лежащих в основе перевода безличных глаголов с библейского иврита на латынь Вульгаты.

Ключевые слова: безличные глаголы, Вульгата, иврит, неканоническая аргументная структура.

Received: 16.08 .2017

Final version received: 11.10 .2017 